\title{
The Effect of Absorbed Dose to Organs at Risk Following Craniospinal Irradiation Using Different Radiotherapy Techniques
}

\author{
Hadeer A. Shahin 1*, Ehab M. Attalla' ${ }^{2}$, H. S. Abou-Elenein ${ }^{3}$, Hussein Elsayed ${ }^{3}$, Ibrahim Bashter ${ }^{1}$, \\ Marwa A. Suliman ${ }^{4}$ \\ ${ }^{1}$ Physics Department, Faculty of Science, Zagazig University, Zagazig, Egypt \\ ${ }^{2}$ Radiotherapy Department, National Cancer Institute, Cairo, Egypt \\ ${ }^{3}$ Radiotherapy Department, Children's Cancer Hospital, Cairo, Egypt \\ ${ }^{4}$ Clinical Oncology \& Nuclear Medicine Department, Faculty of Medicine, Suez Canal University, Ismailia, Egypt \\ Email: *hadeershahin201@gmail.com
}

How to cite this paper: Shahin, H.A., Attalla, E.M., Abou-Elenein, H.S., Elsayed, H., Bashter, I. and Suliman, M.A. (2020) The Effect of Absorbed Dose to Organs at Risk Following Craniospinal Irradiation Using Different Radiotherapy Techniques. International Journal of Medical Physics, Clinical Engineering and Radiation Oncology, 9, 73-85.

https://doi.org/10.4236/ijmpcero.2020.92008

Received: February 14, 2020

Accepted: May 6, 2020

Published: May 9, 2020

Copyright ( 2020 by author(s) and Scientific Research Publishing Inc. This work is licensed under the Creative Commons Attribution International License (CC BY 4.0).

http://creativecommons.org/licenses/by/4.0/ (c) (i) Open Access

\begin{abstract}
Introduction: In medulloblastoma patients craniospinal irradiation is an important element the treatment. Our study aimed to evaluate the effect of absorbed dose to organs at risk using the hybrid intensity-modulated radiation therapy (IMRT) versus three-dimensional conformal radiotherapy (3DCRT) for craniospinal irradiation (CSI) in average risk medulloblastoma patients. Materials and Methods: In this study, thirteen medulloblastoma patients were included. The prescribed total dose to the planning target volume (PTV) was $23,40 \mathrm{~Gy}$ in 13 fractions. Two radiotherapy techniques, three dimensional conformal radiotherapy (3DCRT) and hybrid intensity modulated radiotherapy (IMRT) were used to treat these patients. The coverage of the Target was evaluated using the D mean, D95\%, D2\%, D98\% and V95\%. Other parameters were also compared such as Integral dose (ID), Homogeneity index (HI) and doses to the organs at risk (OARs). Results: There was no significant difference in the mean dose received by the PTV-Brain or the dose received by $95 \%$ and $98 \%$ of PTV volume using the two techniques. For PTV-Spine, the percentage volume receiving $95 \%$ of the total dose increased significantly in the hybrid IMRT technique compared to the conformal technique. So, hybrid IMRT plan achieved the best coverage for PTV spine. Lower dose for OAR was delivered by $3 \mathrm{DCRT}$, except the heart and thyroid, hybrid IMRT achieved better sparing. All plans resulted in the same dose homogeneity index (DHI) for PTV-Brain. For PTV-Spine, hybrid IMRT technique achieved better dose homogeneity compared to 3DCRT technique (1.09 vs. 1.12 ; $\mathrm{p}>0.05)$. Conclusions: hybrid IMRT technique can be realized on conformal technique because it achieved better dose coverage for the (PTV) and organ at risk (OAR).
\end{abstract}


3DCRT reduced mean dose to most OARS, except the heart and thyroid. Therefore, the hybrid IMRT technique may be a CSI treatment alternative to 3DCRT.

\section{Keywords}

Craniospinal Irradiation, Three-Dimensional Conformal Radiotherapy, Intensity-Modulated Radiation Therapy, Medulloblastoma, Organs at Risk, Homogeneity Index

\section{Introduction}

In medulloblastoma and other tumors of central nervous system, Craniospinal radiotherapy is considered a significant technique in the treatment. Medulloblastoma is considered one of the most common brain tumors in children accounts about $20 \%$ - $25 \%$ of all cancer malignancies [1] [2]. Patients with Standard risk are associated with survival rates of $85 \%$ while for high risk patients the survival rates about $70 \%$ [3]. There are different techniques of radiotherapy, such as (3DCRT) and (IMRT), which replaced the traditional large field radiotherapy technology used in treatment of CSI. The target of CSI is considered a large and complex shaped target and that requires complex treatment planning, which usually requires matching a large number of fields and different isocenter to obtain good plans [4] [5]. With continuous improvements in long-term survival, especially average-risk medulloblastoma in children, there are long-term side effects (neurocognitive decline, hearing impairment, growth retardation, endocrine dysfunction, cataract, fertility impairment and second malignancies) [6].

There are many critical factors affected the cranial irradiation, which including the daily fraction, total radiation dose to each organ at risk, patient age during treatment and the combination of other treatment modalities with radiotherapy (Surgery and Chemotherapy) [7]. Pamela et al. recommended that smart arc was superior to using Helical Tomo therapy (HT) as well as 3D-CRT in terms of greater PTV homogeneity, and also achieving reduced maximum doses to many normal tissues [8]. There are multiple studies published on craniospinal irradiation using different delivery methods, the two methods which used in this study have not been compared and analyzed using the same patients and similar optimization criteria [9] [10] [11].

Due to the complexity of conventional radiotherapy techniques based on field junctions, new ways of treatment delivery using more modern IMRT techniques is essential. Better patient outcomes and patient comfort throughout treatment is the important of any course of treatment and it is achieved by advanced methods of treatment. Doses to healthy tissue during radiation therapy can lead to various side effects (early and late effects) and causes a second cancer's risk. For patients surviving more than 10 years, the incidence of second malignancies al- 
most double in IMRT compared with conformal radiotherapy from about $1 \%$ to $1.75 \%$ [12] [13].

The present study aimed to compare the radiation doses delivered to tumor and organs at risk using two different craniospinal radiotherapy techniques and evaluating which technique is more effective and reduce doses to OAR.

\section{Materials and Methods}

Thirteen medulloblastoma patients $(n=13)$ were included in this study (mean age was 7 years; range, 4 - 11 years). Two different CSI treatment plans were designed for every patient, one was 3DCRT and the other was Conventional plus 5IMRT beams (hybrid IMRT). However even with advanced techniques matching of cranial and spinal fields is an unavoidable situation. The two plans generated were compared using the parameters of D mean, D98\%, D95\%, D2\%, V95\%, HI, and doses to the organs at risk (OARs). The thirteen patients were average risk medulloblastoma patients who received (23.4 Gy in 13 fractions).

The setup of all patients was in a supine position. Fixation of the patients done by using thermoplastic mask and a body mask. Scan range was from the top of the head to the proximal femur. Slice thickness was $5 \mathrm{~mm}$.

\subsection{Delineation and Planning}

Delineation of (PTV) and OARs were contoured slice by slice based on CT images according to ICRU (International Commission on Radiation Units and Measurements) recommendations. PTV included the brain (PTV-Brain) and spinal cord (PTV-Spinal). PTV-Brain included the whole brain with margin 5 $\mathrm{mm}$ around the clinical target volume (CTV). PTV-Spinal included C2 through $\mathrm{S} 2$ and $5 \mathrm{~mm}$ beyond their external boundary. Thyroid, eyes, heart, lungs, kidneys and liver, were delineated as OARs for comparison. All patients Plans were generated using Elekta computerized medical systems (CMS) Inc.'s (St. Louis $\mathrm{MO}$ ) $\mathrm{XiO}$ software version 4.4. The linear accelerator used in this study was Siemens ONCOR accelerator (Siemens, Malvern, PA) with an 82-leaf multi-leaf collimator (MLC).The dose calculation was performed using superposition algorithm.

\subsection{Radiotherapy Techniques}

\subsubsection{Three Dimensional Conformal Radiotherapy (3DCRT)}

Conventional 3DCRT plans using $6 \mathrm{MV} \mathrm{X}$-rays on a XiO TPS were generated for each patient. For the PTV-Brain; two opposed lateral fields were used, collimated to match the divergence of the direct posterior spinal field. Bilateral cranial beams and spinal fields were shaped using a Millennium Multi Leaf Collimator (MLC) to confirm the field to the PTV and to shield the critical organs. Dose was prescribed and normalized to the reference point at the geometric center of the PTV-Brain. The spinal field was weighted to achieve better coverage of the PTV-Spine. 


\subsubsection{Hybrid Intensity Modulated Radiotherapy (IMRT)}

This Plan includes two separate plans, one for PTV-Brain and the other for PTV-Spine using $6 \mathrm{MV}$ photons on a XiO TPS. The spinal PTV was first planned using a five-field inverse planning technique with gantry angles of $180^{\circ}$, $45^{\circ}, 315^{\circ}, 130^{\circ}$ and $230^{\circ}$. The isocenter was set at the geometrical center of the PTV-Spine. The $2^{\text {nd }}$ plan was for the PTV-Brain. For the cranial fields the isocenter was set at the center of the PTV. This plan used lateral opposed half-beam blocked fields with a MLC. A uniform margin of $1 \mathrm{~cm}$ was added around the PTV-Brain except for the caudal direction to account for penumbra and to improve target coverage. MLC were used to minimize the dose to the OARs without affecting target coverage. Collimator angles were used to match the dose gradient from the IMRT spine plan. Both spinal and cranial plans were combined to achieve the final plan (3DCRT plus IMRT) for the entire craniospinal axis. Three rings were used around the PTV spine with margin 0.5, 1 and $1.5 \mathrm{~cm}$ respectively to achieve the coverage of PTV spine and minimize the doses to the OARs.

\subsection{Plan Evaluation}

The plans were compared based on target volume coverage, defined as the volume of PTV receiving at least 95\% (V95\%) and 107\% (107\%) of the prescribed dose respectively. The parameters Dmean, D98\%, D95\% and D2\% were also used for plan evaluation, where D mean, D98\%, D2\% and D95\% values are defined as the dose received by $98 \%, 2 \%$ and $95 \%$ of the PTV volume these values are represented the mean, maximum and minimum doses in the PTV, D95\% defined as the dose received by $95 \%$ of the PTV volume. Dose homogeneity, OAR sparing and integral doses (IDs) was also compared. Dose homogeneity was evaluated quantitatively using the dose homogeneity index (DHI), defined as the ratio between the doses to 5\% (D5\%) and 95\% (D95\% of the volume of PTV using the following equation:

$$
\mathrm{HI}=(\mathrm{D} 5 \% / \mathrm{D} 95 \%)
$$

The optimal HI value is 1 .

The ID for the target volumes and various OARs were calculated using the equation:

$$
\mathrm{ID}=\rho^{*} V^{*} D
$$

where $\rho, V$ and $D$ are the density, volume and mean dose of the organ, respectively (12).

\subsection{Statistical Analysis}

Mean and standard deviation values were calculated using Microsoft Excel 2010, while data significance was calculated using Duncan multiple range test in IBM SPSS statistics 20 software packages. A $P$-value is considered statistically significant less than 0.05 . 


\section{Results}

Table 1 illustrates the difference between two techniques for dose-volume parameters derived from DVHs for PTV Brain and PTV Spine. D95\% is the dose delivered to $95 \%$ of the target volume (PTV). V95\% is the PTV volume receiving $95 \%$ of the prescribed dose. P-values were calculated using paired samples $t$ test. The table showed that there is a significant difference between them regarding the D95\% (Gy), D98\% (Gy), D5\% (Gy), V95\% and V107\%.

Coverage: There was no significant difference in the mean dose received by the PTV-Brain or the dose received by $95 \%$ and $98 \%$ of PTV volume. Also, the percentage volume receiving $95 \%$ of the total dose was comparable in the two techniques. For PTV-Spine, the percentage volume receiving 95\% of the total dose increased significantly in the hybrid IMRT techniques compared to the conformal technique $(\mathrm{P}=0.00)$.

Figure 1 and Figure 2 showed that the dose received by $2 \%$ in the 3DCRT, which represent the maximum dose is higher when compared by hybrid IMRT for both PTV-Brain and PTV-Spine. The volume receiving higher dose (V107\%)

Table 1. Comparison between The PTV dose parameters for the two plans.

\begin{tabular}{|c|c|c|c|c|c|c|}
\hline \multirow{2}{*}{ Parameter } & \multicolumn{3}{|c|}{ PTV-Brain } & \multicolumn{3}{|c|}{ PTV-Spine } \\
\hline & 3DCRT & IMRT & P-Value & 3DRT & IMRT & P-Value \\
\hline \multicolumn{7}{|c|}{ PTV Mean. dose: } \\
\hline Mean & 23.40 & 23.41 & 0.7 & 26.16 & 26.22 & 0.9 \\
\hline SD & \pm 0.2 & \pm 0.1 & & \pm 0.4 & \pm 0.8 & \\
\hline \multicolumn{7}{|c|}{ PTV Max dose: } \\
\hline Mean & 26.25 & 25.61 & 0.6 & 23.58 & 23.79 & 0.03 \\
\hline $\mathrm{SD}$ & \pm 0.4 & \pm 0.1 & & \pm 0.2 & \pm 0.3 & \\
\hline \multicolumn{7}{|l|}{ D95\%: } \\
\hline Mean & 94.3 & 94.18 & 0.3 & 94.72 & 97.38 & 0.00 \\
\hline $\mathrm{SD}$ & \pm 0.02 & \pm 0.02 & & \pm 0.01 & \pm 0.01 & \\
\hline \multicolumn{7}{|l|}{ V95\%: } \\
\hline Mean & 94.68 & 94.49 & 0.03 & 94.13 & 98.28 & 0.00 \\
\hline $\mathrm{SD}$ & \pm 1.8 & \pm 1.8 & & \pm 1.9 & \pm 1.6 & \\
\hline \multicolumn{7}{|l|}{ D98\%: } \\
\hline Mean & 89.20 & 88.82 & 0.3 & 92.51 & 95.65 & 0.00 \\
\hline $\mathrm{SD}$ & \pm 0.03 & \pm 0.03 & & \pm 0.01 & \pm 0.01 & \\
\hline \multicolumn{7}{|l|}{ D5\%: } \\
\hline Mean & 109 & 105 & 0.3 & 106 & 106 & 0.8 \\
\hline $\mathrm{SD}$ & \pm 0.2 & \pm 0.01 & & \pm 0.01 & \pm 0.1 & \\
\hline \multicolumn{7}{|l|}{ D2\%: } \\
\hline Mean & 37.21 & 37.96 & 0.4 & 37.73 & 38.96 & 0.1 \\
\hline $\mathrm{SD}$ & \pm 2.04 & \pm 0.2 & & \pm 1.6 & \pm 0.5 & \\
\hline \multicolumn{7}{|l|}{ V107\%: } \\
\hline Mean & 0.066 & 0.35 & 0.1 & 0.5 & 2.26 & 0.004 \\
\hline $\mathrm{SD}$ & \pm 0.2 & \pm 0.6 & & \pm 0.7 & \pm 1.7 & \\
\hline
\end{tabular}




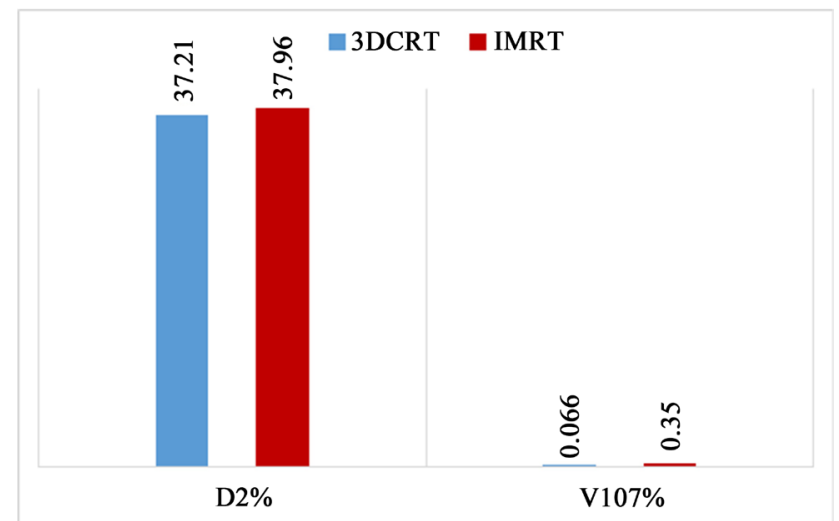

Figure 1. Comparison of V107\% and D2\% for PTV-Brain between the two Planning treatment techniques.

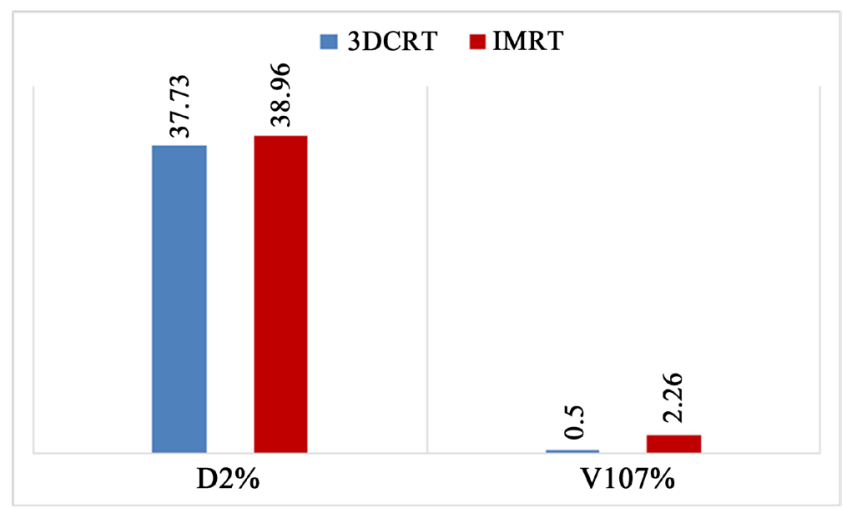

Figure 2. Comparison of V107\% and D2\% for PTV-Spine between the two planning treatment techniques.

of the total dose in the hybrid IMRT increased significantly when compared to the 3DCRT for PTV-Spine $(\mathrm{P}=0.004)$.

\section{Doses to OARs}

Table 2 and Figure 3 showed that the mean dose received by the Rt lung in the 3D-CRT technique was 5.5 Gy compared with $8.1 \mathrm{~Gy}$ in the IMRT. The percentage volume of the Rt lung that received $20 \mathrm{~Gy}$ (V20 Gy) decreased significantly in the hybrid IMRT techniques (2.23\% compared with $7.37 \%$ in 3D-CRT). For the Lt Lung, the mean dose in the 3DCRT technique was 3.68 Gy compared with $7.99 \mathrm{~Gy}$ in the hybrid IMRT. The percentage volume that received 20 Gy (V20 Gy) decrease significantly in the hybrid IMRT technique.

The results showed a significant reduction in the percentage volume of the both lungs in the hybrid IMRT techniques, for both kidneys there is a significant reduction in the mean dose in the 3DCRT techniques ( $\mathrm{p}$-value $=0.00$ ).

The two treatment planning techniques are presented in Figure 4. 3DCRT technique included two opposed lateral cranial photon fields and one posterior spinal photon fields. Hybrid IMRT technique consisted of two opposed lateral cranial photon fields, five inversed posterior photon fields for spinal irradiation. 
Table 2. Doses to the organs at risk.

\begin{tabular}{|c|c|c|c|}
\hline OAR & 3D-CRT & IMRT & P-Value \\
\hline \multicolumn{4}{|l|}{ Rt Lung. } \\
\hline \multicolumn{4}{|l|}{ Mean dose: } \\
\hline Mean & 5.51 & 8.10 & 0.00 \\
\hline $\mathrm{SD}$ & \pm 0.8 & \pm 0.9 & \\
\hline \multicolumn{4}{|l|}{ Rt Lung. } \\
\hline V20: & & & \multirow{3}{*}{0.00} \\
\hline Mean & 7.37 & 2.23 & \\
\hline $\mathrm{SD}$ & \pm 0.2 & \pm 0.1 & \\
\hline \multicolumn{4}{|l|}{ Lt Lung. } \\
\hline \multicolumn{4}{|l|}{ Mean dose: } \\
\hline Mean & 3.68 & 7.99 & \multirow{2}{*}{0.00} \\
\hline $\mathrm{SD}$ & \pm 0.9 & \pm 0.8 & \\
\hline \multicolumn{4}{|l|}{ Lt Lung. } \\
\hline V20: & & & \multirow{3}{*}{0.00} \\
\hline Mean & 3.7 & 1.8 & \\
\hline $\mathrm{SD}$ & \pm 0.2 & \pm 0.1 & \\
\hline \multicolumn{4}{|l|}{ Rt Kidney. } \\
\hline \multicolumn{4}{|l|}{ Mean dose: } \\
\hline Mean & 4.8 & 8.03 & \multirow{2}{*}{0.00} \\
\hline $\mathrm{SD}$ & \pm 0.2 & \pm 0.1 & \\
\hline \multicolumn{4}{|l|}{ Lt Kidney. } \\
\hline \multicolumn{4}{|l|}{ Mean dose: } \\
\hline Mean & 4 & 7.9 & \multirow{2}{*}{0.00} \\
\hline $\mathrm{SD}$ & \pm 0.2 & \pm 0.1 & \\
\hline \multicolumn{4}{|l|}{ Liver. } \\
\hline \multicolumn{4}{|l|}{ Mean dose: } \\
\hline Mean & 5.69 & 6.65 & 0.00 \\
\hline $\mathrm{SD}$ & \pm 0.7 & \pm 0.7 & \\
\hline \multicolumn{4}{|l|}{ Heart. } \\
\hline \multicolumn{4}{|l|}{ Mean dose: } \\
\hline Mean & 12.7 & 6.82 & 0.00 \\
\hline $\mathrm{SD}$ & \pm 1.8 & \pm 0.8 & \\
\hline \multicolumn{4}{|l|}{ Thyroid. } \\
\hline \multicolumn{4}{|l|}{ Mean dose: } \\
\hline Mean & 18.60 & 17.41 & 0.07 \\
\hline $\mathrm{SD}$ & 0.9 & \pm 0.3 & \\
\hline \multicolumn{4}{|l|}{ Rt Eye. } \\
\hline \multicolumn{4}{|l|}{ Mean dose: } \\
\hline Mean & 21.22 & 21.62 & 0.2 \\
\hline $\mathrm{SD}$ & \pm 1.7 & \pm 1.56 & \\
\hline \multicolumn{4}{|c|}{ Lt Eye. Mean dose: } \\
\hline Mean & 21.02 & 21.07 & 0.9 \\
\hline $\mathrm{SD}$ & \pm 1.8 & \pm 2.01 & \\
\hline
\end{tabular}




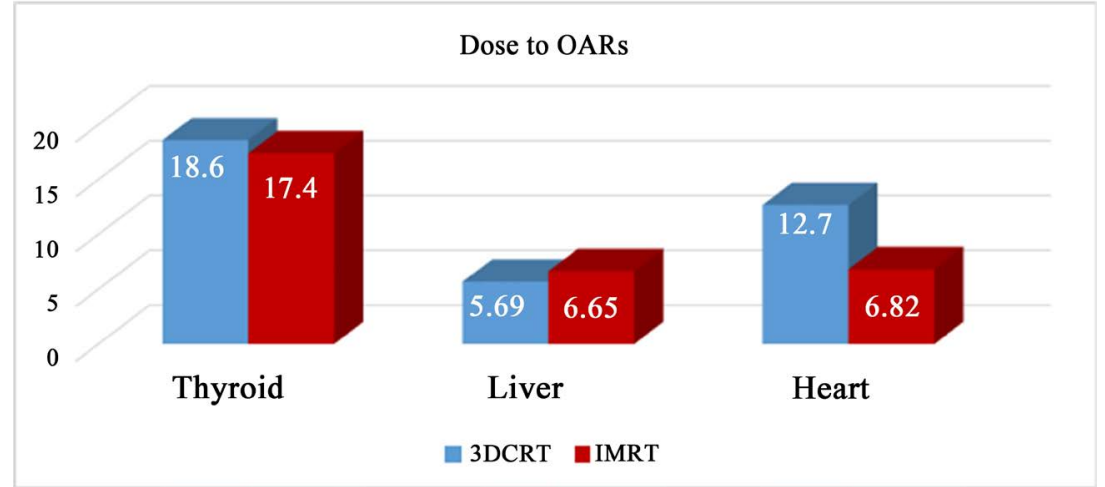

Figure 3. Represents comparison of Mean dose of the heart, liver and thyroid between the two planning techniques.

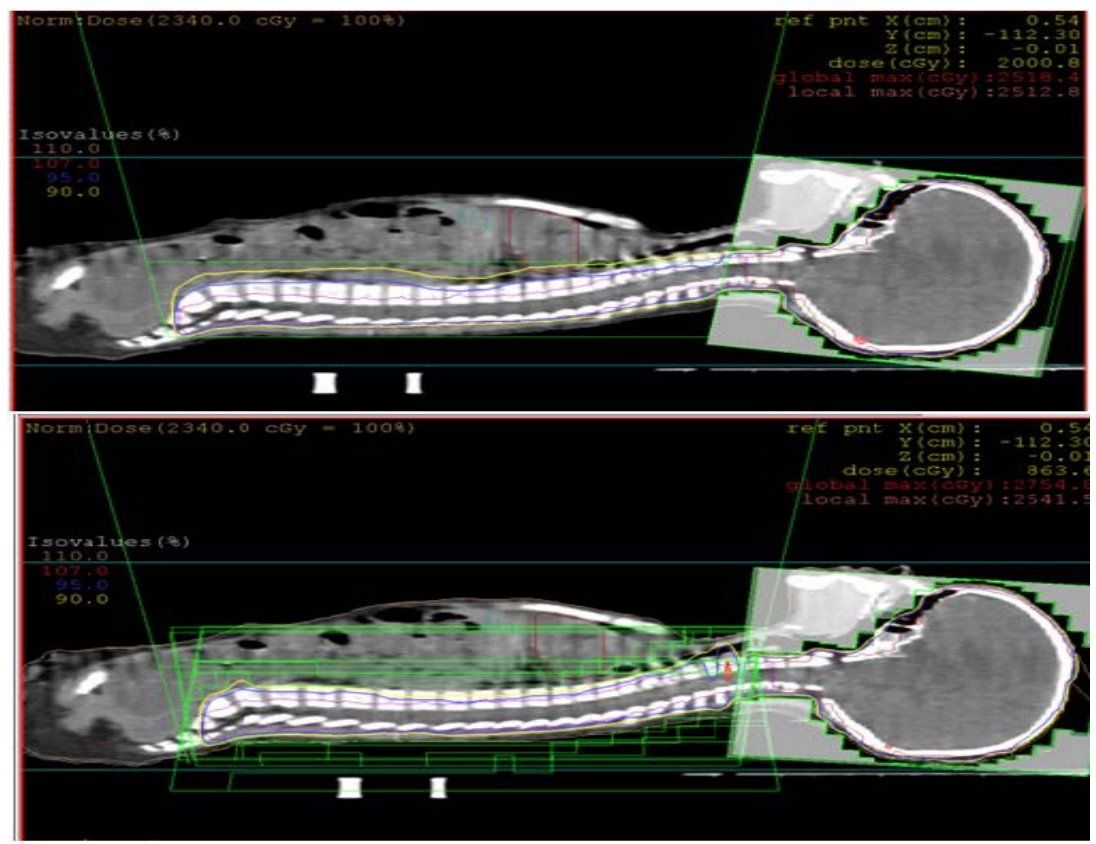

Figure 4. The sagittal dose distribution curves from the two techniques. The blue color shows $95 \%$ isodose line.

IMRT plan achieve highly conformal doses compared with 3DCRT.

Figure 5 and Figure 6 chart of the HI for the PTV-Brain and PTV-Spine for each technique. IMRT plans achieved the lowest mean HI compared with 3DCRT plans for PTV-Spine, while the HI was comparable for PTV-Brain.

Table 3 Illustrate the results of the integral doses calculated for thirteen patients for each treatment method. On average, the two techniques have comparable integral dose values. 3D-CRT provided the lowest average integral dose values while IMRT plans showed the highest overall values.

\section{Discussion}

Two different methods for treatment of CSI of medulloblastoma patients were used in this study, 3DCRT in comparison with hybrid IMRT. The main advantage 


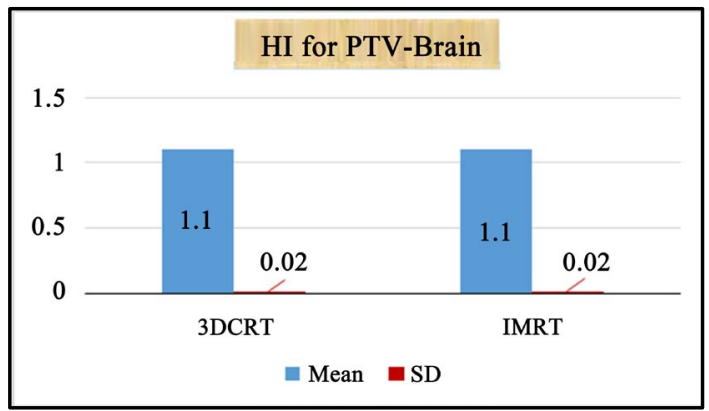

Figure 5. Mean and SD of HI for the PTV-Brain using the two techniques.

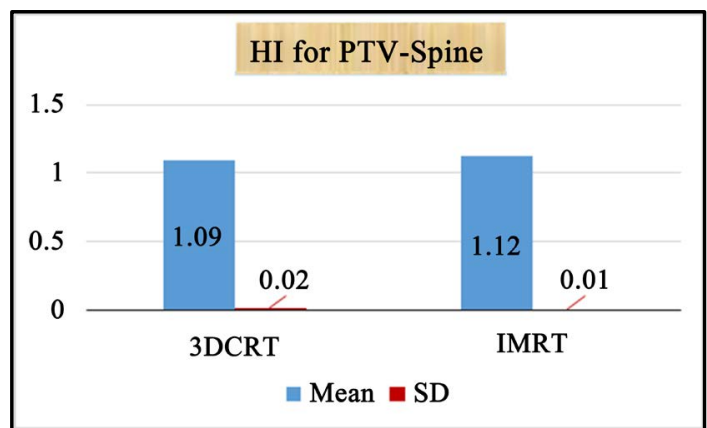

Figure 6. Mean and SD of HI for the PTV-Spine using the two techniques.

Table 3. Integral dose to the various organs at risk.

\begin{tabular}{ccccccc}
\hline \multirow{2}{*}{ Volumes } & \multirow{2}{*}{$\begin{array}{c}\text { Density } \\
\left(\mathrm{g} \cdot \mathrm{cm}^{-3}\right)\end{array}$} & $\begin{array}{c}\text { Volume } \\
\left(\mathrm{cm}^{3}\right)\end{array}$ & \multicolumn{2}{c}{ Mean dose(Gy) } & \multicolumn{2}{c}{ Integral dose (Gy Kg) } \\
\cline { 6 - 7 } Rt Lung & 0.5 & 413.5 & 5.5 & 8.1 & 1.1 & 1.7 \\
Lt Lung & 0.5 & 405 & 5.3 & 7.9 & 1.1 & 1.6 \\
Rt Kidney & 1.05 & 70.4 & 4.8 & 8.03 & 0.3 & 0.6 \\
Lt Kidney & 1.07 & 72.4 & 4.00 & 7.93 & 0.3 & 0.6 \\
Liver & 1.05 & 674 & 5.79 & 6.65 & 4.1 & 4.7 \\
Heart & 1.08 & 203.9 & 12.7 & 6.82 & 2.7 & 1.5 \\
Thyroid & 1.09 & 5.3 & 18.96 & 17.42 & 0.1 & 0.1 \\
Lt eye & 0.9 & 7.9 & 21.23 & 21.07 & 0.1 & 0.1 \\
Rt eye & 1.01 & 7.8 & 21.02 & 21.62 & 0.2 & 0.2 \\
\hline
\end{tabular}

behind exploring IMRT for medulloblastoma has been conformal the dose to the target and for better sparing of OAR. Patients with supine position has been assessed while delivering better doses to spinal cord and also saving setup time [8] [14] [15] [16].

In our study, the average values of D95\%, V95\%, mean dose and D2\% associated with DVH using two techniques were $94.3 \%$ vs $94.7 \%, 94.7 \%$ vs $94.4 \%$, 23.4 Gy vs 23.4 Gy and 37.2 Gy vs 37.9 Gy respectively, for PTV brain. These values were $94.7 \%$ vs $97.4 \%, 94.1 \%$ vs $98.3 \%, 26.2$ Gy vs 26.2 Gy and 37.7 Gy vs 38.9 Gy respectively, for PTV spine. From these results we can notice that max- 
imum dose inside target volume increased (increasing in D2\% values) using hybrid IMRT technique. At the same time, the target coverage increasing using hybrid IMRT technique (V95\% and D95\% values).

These results are in agreement with the results reported by Sharma et al., who showed in their study that IMRT for CSI can be realized on a conventional linear accelerator [7]. Also, recently Parker et al., have reported the feasibility of conventional linear accelerator based intensity-modulated radiotherapy (IMRT) for CSI in small children. However, even with this advanced technique, matching of cranial and spinal fields is an unavoidable situation in LA-based IMRT for CSI [17].

Our results showed that the IMRT plans have best coverage of PTV with the prescribed dose. However; the OARs are better spared with the 3DCRT compared to hybrid IMRT while achieved better sparing for the heart and thyroid. Also there was significant reduction in the dose to OARs with hybrid IMRT especially for the organ that is away from the tumor. The PTV coverage was acceptable if $95 \%$ of the volume were covered by $95 \%$ of the prescribed doses.

Hybrid IMRT radiotherapy achieved best dose homogeneity in comparison with 3DCRT as shown in Figure 5 and Figure 6 the dose homogeneity was comparable for PTV-brain while hybrid IMRT achieved better dose HI compared with 3DCRT (1.09 vs. 1.12). Using of five-field inversely planned IMRT for the spinal target volume achieved an improvement in the dose homogeneity and reduction of OAR doses when compared with 3DCRT especially in thyroid, these results were in agreement with the results report by Parker et al. [17]. While our mean value of V107\% was slightly lesser in the 3DCRT plan it is high in the hybrid IMRT plan for both PTV brain and PTV spine. Kunos et al., investigated the role of Tomo Therapy based CSI in limiting dose to growing vertebrae in four pediatric patients with medulloblastoma and compared it with conventional techniques. Helical TomoTherapy provided improved dose avoidance to growing vertebrae when compared with conventional [18].

We also revealed in our study that the integral dose calculations for each patient and each treatment method as well as the overall average for each treatment method. These whole body integral doses were tabulated using a normal tissue volume of the patient, taken to be from the vertex to about $5 \mathrm{~cm}$ below the end of the spinal cord, and the average density of this volume. The results revealed that the whole body integral dose is comparable for the two treatment techniques 3DCRT and hybrid IMRT. The findings here are similar to those found by Penagaricano, et al. [19] who recommended that there is no difference between techniques. In this study the beam geometry and MLC shaping used for PTV brain was identical in the two techniques. This was done to avoid complexity that would arise if a multi field IMRT plan was generated for the brain to match dosimetrically with the spinal IMRT plan.

Several authors founded that IMRT increase integral dose to OARs and therefore, risk of secondary malignancies increased compared with 3DCRT [19] 
[20]. The main purpose behind exploring IMRT for CSI has been better sparing of normal tissue such as thyroid. In fact, IMRT technique better than conformal radiotherapy for PTV even for spinal lengths exceeding the maximum allowable field sizes while the junction and feathering will be neglected. Even the IMRT technique is easier; larger volume is received lower doses because the total MUs in IMRT is higher than that of 3D-CRT where the additional radiation dose to normal tissues with IMRT has been increased the probability of induce another primary cancer IMRT than in 3D-CRT.

Finally, in this study there are some limitations. First, difference between the gender and age may be present. Moreover, due to the limited algorithm in XIO planning system, limitations in estimating IMRT plan must be noted.

So, althoughLinac-3DCRT is the best in terms of resource distribution because of its simple planning and treatment time, these benefits do not overcome problems in dose homogeneity, NTID and organ sparing. Therefore, the clinics with only Linacs, it may be worth exploring other advanced methods for CSI treatments, such as volumetric-modulated arc therapy (VMAT) while VMAT offers a new treatment method with decreased ID while offering comparable normal tissue sparing to Tomo Helical radiotherapy [21]. However, this technique does not address the field-size limitation of a Linac, still necessitating junction matching between the cranial and spinal fields, and between two spinal fields for longer PTVs.

\section{Conclusions}

CSI still one of the most challenging processes in radiation planning, delivery and verification. IMRT technique preferred to plan such complex shaped and long target volumes, while field matching, junction will be neglected.

Hybrid IMRT technique for CSI is favorable while showed larger effect for PTV-Spine than the PTV-Cranium.

Hybrid IMRT technique achieved slightly better PTV homogeneity, and was noted to have reductions in mean dose of heart and thyroid when compared to 3DCRT technique, also, reduction of integral dose in non-target tissue.

Because of the longer time in hybrid IMRT plans the probability to induce second primary cancer is higher than in 3DCRT. So, in the future: extensive research is needed to evaluate the clinical implications of these findings in the reduction of treatment toxicities and secondary malignancies.

\section{Acknowledgements}

I would like to thank all the staff in the Department of Radiation therapy of 57357 and Special thanks to Dr. Engy Salah, Med. Phy. Hamdy Ismail, Med. Phy. Moataz Ahmed and Med. Phy. Samar Emad for their help.

\section{Conflicts of Interest}

The authors declare no conflicts of interest regarding the publication of this paper. 


\section{References}

[1] Lopez Guerra, J.L., Marrone, I. and Jaen, J. (2014) Outcome and Toxicity Using Helical Tomotherapy for Craniospinal Irradiation in Pediatric Medulloblastoma. Clinical and Translational Oncology, 16, 96-101. https://doi.org/10.1007/s12094-013-1048-7

[2] Berry, M.P., Jenkin, R.D., Keen, C.W., et al. (1981) Radiation Treatment for Medulloblastoma: A 21-Year review. Journal of Neuroscience, 55, 43-51. https://doi.org/10.3171/jns.1981.55.1.0043

[3] Bauman, G., Yartsev, S., Coad, T., et al. (2005) Helical Tomotherapy for Craniospinal Radiation. The British Journal of Radiology, 78, 548-552. https://doi.org/10.1259/bjr/53491625

[4] Verellen, D. and Vanhavere, F. (1999) Risk Assessment of Radiation-Induced Malignancies Based on Whole-Body Equivalent Dose Estimates for IMRT Treatment in the Head and Neck Region. Radiotherapy and Oncology, 53, 199-203.

https://doi.org/10.1016/S0167-8140(99)00079-1

[5] Packer, R.J., Goldwein, J., Nicholson, H.S., et al. (1999) Treatment of Children with Medulloblastomas with Reduced-Dose Craniospinal Radiation Therapy and Adjuvant Chemotherapy: A Children's Cancer Group Study. Journal of Clinical Oncology, 17, 2127-2136. https://doi.org/10.1200/JCO.1999.17.7.2127

[6] Hughes, E.N., Shillito, J., Sallan, S.E., et al. (1988) Medulloblastoma at the Joint Center for Radiation Therapy between 1968 and 1984. The Influence of Radiation Dose on the Patterns of Failure and Survival. Cancer, 61, 1992-1998. https://doi.org/10.1002/1097-0142(19880515)61:10<1992::AID-CNCR2820611011> 3.0.CO;2-J

[7] Sharma, D.S., Gupta, T., Jalali, R., et al. (2009) High-Precision Radiotherapy for Craniospinal Irradiation: Evaluation of Three-Dimensional Conformal Radiotherapy, Intensity-Modulated Radiation Therapy and Helical Tomotherapy. The British Journal of Radiology, 82, 1000-1009. https://doi.org/10.1259/bjr/13776022

[8] Myers, P., Stathakis, S., Gutiérrez, A.N., et al. (2013) Dosimetric Comparison of Craniospinal Axis Irradiation (CSI) Treatments Using Helical Tomotherapy, Smartarc ${ }^{\mathrm{TM}}$ and 3D Conventional Radiation Therapy. International Journal of Medical Physics, Clinical Engineering and Radiation Oncology, 2, 30-38. https://doi.org/10.4236/ijmpcero.2013.21005

[9] Davidg, K.I.R., Nancy, S.C.H., Tarbell, J., et al. (2004) Conformal Radiation Therapy for Childhood CNS Tumors. The Oncologist, 9, 442-450.

https://doi.org/10.1634/theoncologist.9-4-442

[10] Evangeline, S.Q., et al. (2017) A Review of Dosimetric and Toxicity Modeling of Proton versus Photon Craniospinal Irradiation for Pediatrics Medulloblastoma. Acta Oncologica, 56, 1031-1042. https://doi.org/10.1080/0284186X.2017.1324207

[11] Zhang, X., Penagaricano, J., Han, E.Y., et al. (2014) Dosimetric Comparison of Craniospinal Irradiation Using Different Tomotherapy Techniques. Technology in Cancer Research \& Treatment, 14, 440-446. https://doi.org/10.1177/1533034614556932

[12] Paganetti, H. (2012) Assessment of the Risk for Developing a Second Malignancy from Scatterd and Secondary Radiation in Radiation Therapy. Health Physics Society, 103, 652-661. https://doi.org/10.1097/HP.0b013e318261113d

[13] Attalla, E.M., Elnaggar, M.A., et al. (2016) Evaluation of the Second Cancer's Risk in Conformal Therapy and Intensity Modulated Radiotherapy for the Organs inside the Primary Radiation Fields. IOSR Journal of Applied Physics, 8, 25-33. 
[14] Fogliata, A., Bergström, S., Cafaro, I., et al. (2011) Cranio-Spinal Irradiation with Volumetric Modulated Arc Therapy: A Multi-Institutional Treatment Experience. Radiotherapy and Oncology, 99, 79-85. https://doi.org/10.1016/j.radonc.2011.01.023

[15] Yoon, M., Shin, D.H., Kim, J., et al. (2011) Craniospinal Irradiation Techniques: A Dosimetric Comparison of Proton Beams with Standard and Advanced Photon Radiotherapy. International Journal of Radiation Oncology, Biology, Physics, 81, 637-646. https://doi.org/10.1016/j.ijrobp.2010.06.039

[16] Mavroidis, P., Ferreira, B.C., Shi, C., Delichas, M.G., et al. (2009) Comparison of the Helical Tomotherapy and MLC-Based IMRT Radiation Modalities in Treating Brain and Cranio-Spinal Tumors. Technology in Cancer Research \& Treatment, 8, 3-14. https://doi.org/10.1177/153303460900800102

[17] Parker, W., Brodeur, M., Roberge, D., et al. (2010) Standard and Nonstandard Craniospinal Radiotherapy Using Helical Tomotherapy. International Journal of Radiation Oncology, Biology, Physics, 77, 926-931. https://doi.org/10.1016/j.ijrobp.2009.09.020

[18] Kunos, C.A., Dobbins, D.C., Kulasekere, R., et al. (2008) Comparison of Helical Tomotherapy versus Conventional Radiation to Deliver Craniospinal Radiation. Technology in Cancer Research \& Treatment, 7, 227-233. https://doi.org/10.1177/153303460800700308

[19] Penagaricano, J.A., Papanikolaou, N., Yan, Y., Youssef, E., et al. (2005) Feasibility of Cranio-Spinal Axis Irradiation with the Hi-Art Tomotherapy System. Radiotherapy and Oncology, 76, 72-78. https://doi.org/10.1016/j.radonc.2005.06.013

[20] Hall, E.J. and Wuu, C.S. (2003) Radiation-Induced Second Cancers: The Impact of 3D-CRT and IMRT. International Journal of Radiation Oncology, Biology, Physics, 56, 83-88. https://doi.org/10.1016/S0360-3016(03)00073-7

[21] Patel, S., Drodge, S., Jacques, A., et al. (2015) A Comparative Planning Analysis and Integral Dose of Volumetric Modulated Arc Therapy, Helical Tomotherapy, and Three-Dimensional Conformal Craniospinal Irradiation for Pediatric Medulloblastoma. Journal of Medical Imaging and Radiation Sciences, 46, 134-140. https://doi.org/10.1016/j.jmir.2014.11.003 